\title{
Hypothetical toroidal, cylindrical, and helical analogs of $\mathrm{C}_{60}$
}

\author{
Chern Chuang, Bih-Yaw Jin* \\ Department of Chemistry, Center of Theoretical Sciences, and Center for Quantum Science and Engineering, National Taiwan University, Taipei 10617, Taiwan, ROC
}

\section{A R T I C L E I N F O}

\section{Article history:}

Received 4 May 2009

Received in revised form 17 July 2009

Accepted 22 July 2009

Available online xxx

\section{PACS:}

85.65.+h

Keywords:

Toroidal

Helical

Carbon nanotube

$\mathrm{C}_{60}$

\begin{abstract}
A B S T R A C T
Toroidal, cylindrical, and helical analogs of $\mathrm{C}_{60}$ buckyball are theoretically constructed and analyzed. In these structures, pentagons and heptagons are separated compactly by hexagons in analogy to pentagons in $C_{60}$ and heptagons in $C_{168}$ proposed by Vanderbilt and Tersoff (1992) [2]. Specifically, all nonhexagons therein are surrounded by hexagons and hexagons are surrounded alternatively by hexagons and nonhexagons, i.e. these structures are polyhedra of Clar type with all their Clar rings nonhexagonal. Quantum chemical calculations have been carried out which show that they possess stabilities comparative to that of $C_{60}$. And their structural features are also investigated in detail. Buckled carbon nanotubes deriving from buckytori with periodically varying radii are suggested to be candidacies for the product of coalescing arrays of $C_{60}$. The helicity of the buckyhelices as a function of their characterizing shifting parameters is studied. In the limit of large shifting parameter, the buckyhelices adopt an unusual geometric form that has not been reported in the literature yet.
\end{abstract}

(c) 2009 Elsevier Inc.. All rights reserved.

\section{Introduction}

In the early nineties, right after the discovery of carbon nanotubes (CNT) [1], Vanderbilt and Tersoff [2] proposed a negative-curvature analog of $\mathrm{C}_{60}$, which is basically a triply periodic $\mathrm{C}_{168}$ structure composed of only hexagons and heptagons. While the pentagons in $\mathrm{C}_{60}$ are all surrounded by hexagons and the hexagons are surrounded by alternating hexagons and pentagons, the heptagons in $\mathrm{C}_{168}$ are surrounded by hexagons and the hexagons are surrounded by alternating hexagons and heptagons. The resulting structure for $\mathrm{C}_{168}$ is like replacing each carbon atom in a diamond lattice with hollowed carbon nanotetrapod (superatom), and the bonding between these superatoms with a suitable nanotube (superbond). Topologically speaking, $C_{60}$ is isomorphic to a sphere and $\mathrm{C}_{168}$ is isomorphic to the D-type triply periodic minimal surface (TPMS) [3-7]. According to the GaussBonnet theorem, each unit cell in $C_{168}$ contains exactly 24 heptagons and every carbon atom in this structure belongs to a particular heptagon. This amazing analogy between the two molecular structures raises an immediate question: Is it possible to construct a graphitic structure, which may possess both positive and negative Gaussian curvatures at the same time, such that nonhexagons are completely surrounded by hexagons and hexagons are surrounded alternately by hexagons and nonhexagons? In the discussion made by Fowler and Pisanski [8], this is

\footnotetext{
* Corresponding author.

E-mail address: byjin@ntu.edu.tw (B.-Y. Jin).
}

equivalent to asking for Clar type fullerenes with all their Clar rings nonhexagonal, where $\mathrm{C}_{60}$ and $\mathrm{C}_{168}$ are both solutions possessing positive and negative curvatures throughout, respectively.

In this paper, we provide a positive answer to the question. Three different solutions are suggested where they have the same shapes and topologies of a torus, a cylinder, and a helical tube, respectively. The latter two are singly periodic infinite structures and are derived from the torus case. All three graphitic structures proposed have pentagons and heptagons in equal numbers, and each pair of pentagon and heptagon comes together with four hexagons. In other words, the ratio between the numbers of polygons in these structures are $N_{5}: N_{6}: N_{7}=1: 4: 1$, where $N_{i}$ is the number of $i$-gons. Quantum chemical calculations showed that the heat of formation of the toroidal and cylindrical structures are comparable to that of $\mathrm{C}_{60}$. The structural properties and general geometric features of the three molecular structures are discussed.

\section{Buckytori}

It is well known that any toroidal carbon nanotube (TCNT) with all faces hexagonal can be formed by bending, and connecting the ends, of a finite straight carbon nanotube. The polyhex TCNT has been of theoretical interest especially on its magnetic response because of its curious topology and geometry [9-23]. It was found that for certain magic numbers of chiral vectors and the number of unit cells the polyhex TCNT may carry extremely large paramagnetic persistent current under an external magnetic field [9]. However, for the polyhex TCNTs to be stable without seriously distorting chemical bondings therein, the number of carbon atoms 
(a)

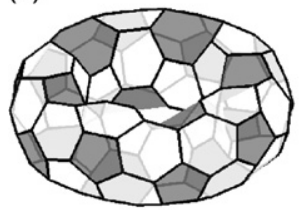

(b)

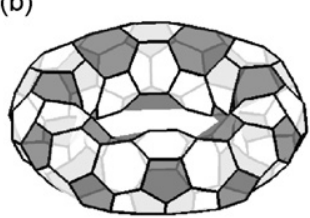

(c)

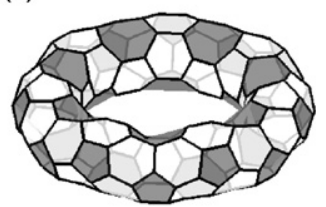

(d)

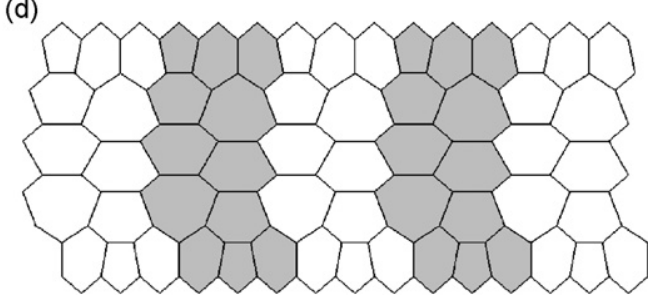

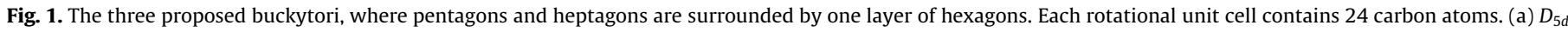
$C_{120}$. (b) $D_{6 d} C_{144}$. (c) $D_{7 d} C_{168}$. (d) The face representation of unfolded $D_{5 d} C_{120}$, where the top and the bottom rows of atoms are repeated.

must be as large as thousands or more so each $\sigma$ bond bears relatively small distortion. Experiments reporting the existence of giant ring-like TCNTs have been carried out [24,25], which are possible candidates of polyhex TCNTs.

Though not yet experimentally confirmed, quantum chemical calculation shows that TCNTs with nonhexagonal defects can be stable molecules, even if they contain only about a hundred of carbon atoms [26-31]. This is because by properly inserting pentagons (heptagons) at the outer-rim (inner-rim) of the torus, the deviations of bond lengths and bond angles from planar graphite are greatly reduced. A very general systematic construction and classification method for TCNTs with nonhexagonal rings that includes most of the existing construction methods for defectcontaining TCNTs as a subset [29-34] was proposed by the present authors [35].

By carefully examining the topology and connectivity of these TCNTs, we found that one particular family of TCNTs precisely satisfies all requirements raised in the previously proposed question. These TCNTs belong to the $D_{n d}$ point group, where $n$ is the rotational symmetry number. In Fig. 1(a)-(c), we show three particular TCNTs with $n=5,6,7$ cases, and the planar graph shown in part (d) corresponds to the unfolded TCNT with $n=5$ based on its face representation. Each of the rotational unit cells contains 24 carbon atoms, so the resulting $D_{5 d}, D_{6 d}$, and $D_{7 d}$ TCNTs contain 120 , 144 , and 168 atoms, respectively. It is interesting to note that the number of carbon atoms in a $D_{7 d}$-TCNT is the same as that in a single unit cell of $C_{168}$. As stated in the question and shown clearly by the three TCNTs in Fig. 1, the pentagons and heptagons are surrounded by hexagons while the hexagons, depending on their loci, are surrounded alternately by three hexagons plus three heptagons, or two heptagons and one pentagons, or one heptagons and two pentagons. In analogy to $\mathrm{C}_{168}$, we deliberately call these TCNTs "buckytori", since they are the realization of buckyball on a torus. As discussed by Vanderbilt and Tersoff, $\mathrm{C}_{60}$ and $\mathrm{C}_{168}$ are derived from the inflation transformation of $\mathrm{C}_{20}$ (dodecahedron) and $\mathrm{C}_{56}$ parent molecules. The three TCNTs shown in Fig. $1, \mathrm{C}_{120}$, $\mathrm{C}_{144}$, and $\mathrm{C}_{168}$, can also be deflated to one third smaller molecules containing only $2 n$ pairs of pentagons and heptagons. The deflation process in these cases can be seen as removing all the hexagons out of the parent structures or, as commented by Vanderbilt and Tersoff, as replacing each hexagon by a single carbon atom at the center of it.

The optimized positions of inequivalent atoms of the total five buckytori, with rotational symmetry number ranging from four to eight, based on the semi-empirical AM1 quantum chemical calculation [36] are given in Table 1 . Since these TCNTs are $D_{n d^{-}}$ symmetric, we only report the coordinates of seven inequivalent atoms in the table. By applying the reflection with respect to $y z$ plane, one can obtain the other five coordinates for atoms with negative $x$ values. The $C_{2}$ rotation about $(\cos (2 \pi / n), \sin (2 \pi / n), 0)$ can generate all 24 coordinates in a single unit cell. Finally the $C_{n}$ rotation about $z$-axis will generate the total $24 n$ atoms of the molecules.

In Table 2, we present some of the structural and physical information of these TCNTs using the AM1 method. It can be seen that the heat of formation per atom of the buckytori, depend on the rotational symmetry number, are comparable to that of $\mathrm{C}_{60}$, although the deviations of bond lengths and bond angles from graphite are a little bit higher. The stabilities of general fullerenes can be explained by a continuum elastic theory [37-43], where the formation energies of curved graphitic structures are the summation of strain energy of bending planar graphene and the local energies of defects. The strain energy term is proportional to integration of the square of mean curvature over the surface of the structure. Since the buckytori possess curvature comparable to that of $\mathrm{C}_{60}$ meanwhile contain more nonhexagonal defects than

Table 1

AM1-optimized coordinates of inequivalent atoms of TCNT $C_{120}, C_{144}$, and $C_{168}$. See text for the generating method of the whole molecule.

\begin{tabular}{|c|c|c|c|c|c|c|c|c|c|}
\hline \multirow[t]{2}{*}{ Atom } & \multicolumn{3}{|l|}{$C_{120}$} & \multicolumn{3}{|l|}{$C_{144}$} & \multicolumn{3}{|l|}{$C_{168}$} \\
\hline & $x$ & $y$ & $z$ & $x$ & $y$ & $z$ & $x$ & $y$ & $z$ \\
\hline 1 & 0.651 & -2.917 & 0.685 & 0.656 & -3.605 & 0.688 & 0.664 & -4.344 & 0.666 \\
\hline 2 & 1.429 & -3.125 & 1.915 & 1.514 & -3.998 & 1.808 & 1.569 & -4.860 & 1.703 \\
\hline 3 & 1.353 & -4.575 & 2.097 & 1.343 & -5.435 & 1.990 & 1.334 & -6.284 & 1.890 \\
\hline 4 & 0 & -5.168 & 1.792 & 0 & -6.024 & 1.709 & 0 & -6.875 & 1.625 \\
\hline 5 & 0.769 & -5.543 & -1.548 & 0.747 & -6.469 & -1.528 & 0.733 & -7.364 & -1.537 \\
\hline 6 & 1.227 & -5.943 & -0.214 & 1.197 & -6.983 & -0.223 & 1.167 & -7.960 & -0.255 \\
\hline 7 & 0 & -5.949 & 0.624 & 0 & -6.929 & 0.631 & 0 & -7.859 & 0.617 \\
\hline
\end{tabular}


Table 2

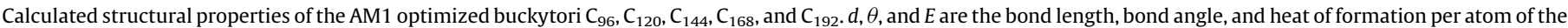

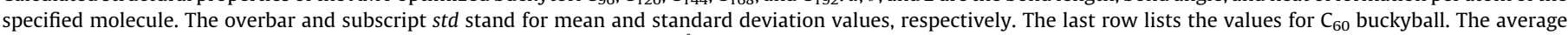
unsigned errors of bond lengths and angles of AM1 calculations are $0.13 \AA$ and $8.77^{\circ}$, respectively.

\begin{tabular}{|c|c|c|c|c|c|}
\hline & $\bar{d}(\AA)$ & $d_{\text {std }}(\AA)$ & $\bar{\theta}\left({ }^{\circ}\right)$ & $\theta_{\text {std }}\left({ }^{\circ}\right)$ & $E(\mathrm{eV})$ \\
\hline $\mathrm{C}_{96}$ & 1.473 & 0.072 & 115.2 & 14.3 & 1.60 \\
\hline $\mathrm{C}_{120}$ & 1.453 & 0.054 & 115.9 & 9.0 & 1.17 \\
\hline $\mathrm{C}_{144}$ & 1.446 & 0.048 & 116.2 & 7.5 & 1.00 \\
\hline $\mathrm{C}_{168}$ & 1.444 & 0.045 & 116.2 & 6.9 & 0.94 \\
\hline$C_{192}$ & 1.435 & 0.045 & 116.2 & 6.9 & 0.94 \\
\hline$C_{60}$ & 1.438 & 0.037 & 116.0 & 5.7 & 0.70 \\
\hline
\end{tabular}

$\mathrm{C}_{60}$, it is reasonable to expect that the buckytori have greater heat of formation per atom than $\mathrm{C}_{60}$.

\section{One-dimensional analogs of $\mathbf{C}_{60}$}

The buckytori discussed above are zero-dimensional finite molecular cages. One may further construct one-dimensional (1D) periodic graphitic structure with the same local geometry by flipping a TCNT inside out and using it as a building block of the periodic 1D structure as shown in Fig. 2. The structure as a whole possesses cylindrical tubular shape with rings of pentagons and heptagons occurring alternately along the direction of periodicity. The radius of this tube varies periodically as one moves along its central axis with the same periodicity as the appearance of the rings of nonhexagons, where its maxima and minima correspond

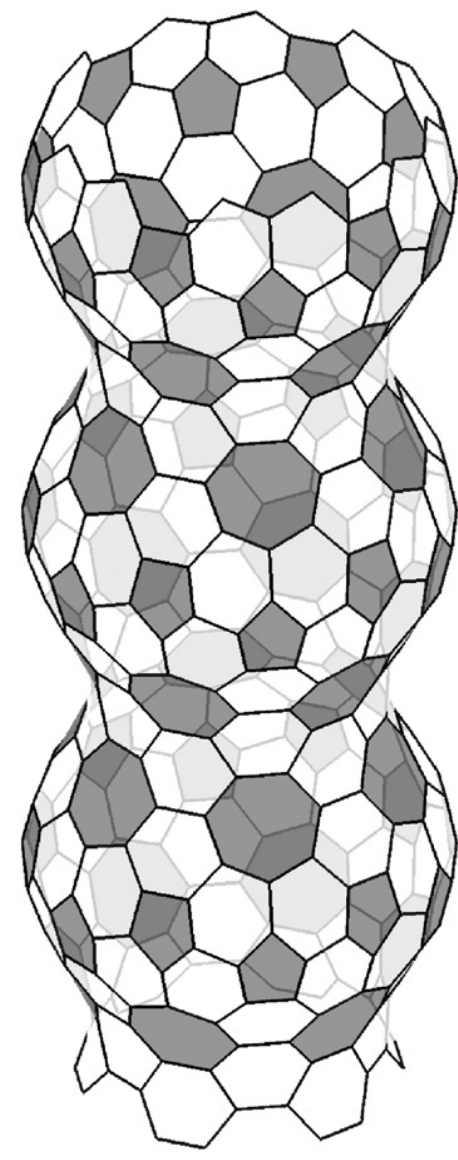

Fig. 2. Buckled carbon nanotube. Three unit cells are shown. The tube shown possesses sixfold rotational symmetry, as its unit cells are obtained by flipping the $D_{6 d} C_{144}$ buckytorus inside out. Similar to its parent molecule, the buckytorus, the buckled tube shares the same local geometry, the tube radius reaches maxima at the altitudes of pentagons and is minimized at the altitudes of heptagons. to the altitudes of pentagons and heptagons, respectively. The second derivative of tube radius with respect to altitude $\left(\partial^{2} r / \partial z^{2}\right)$ and the inverse of radius $(1 / r)$ are the two principal curvatures of the tube. It can be clearly seen that the tube is positively (negatively) curved around the loci of pentagons (heptagons), as the parent TCNT is. Interestingly, when the rotational symmetry number is three, the structure $\mathrm{f}$ this "buckled tube" can also be viewed as an array of $C_{60}$ fused along their $C_{3}$ rotation axes with twelve extra carbon atoms per unit cell, which is the so-called "coalesced" or "corrugated" buckyballs. In particular, we note that Rodriguez-Manzo et al. $[44,45]$ had studied the magnetic properties of similar structures which are the toroidal version of these buckled tubes, formed by bending this "buckled tube" and connecting its two ends.

Here we report the optimized structures and the heat of formation for the buckled CNT with rotational symmetry number ranging from two to eleven, as shown in Tables 3 and 4 . It is interesting to note that, in general, the heat of formation per atom for buckled CNT is much smaller than that of buckytorus with the same rotational symmetry. And the dependence of stability on rotational symmetry number is smoother than in the case of the toroidal isomers. In the hindsight, this result can be rationalized since the condition of asking the atoms in the outer part of a buckytorus to distribute as even as ones in the inner part, and vice versa, is much harder than that of asking homogeneousness of onelayer structures like the buckled CNT. In Fig. 3, the mean radii and the translational vectors of the calculated buckled CNTs are shown. The mean radius is linearly proportional to rotational symmetry number. Whereas the translational vectors decrease monotonically and slowly with increasing rotational symmetry number.

As discussed in our previous report [35], stable helical carbon nanotubes (HCNT) can be formed by distorting and dissecting a

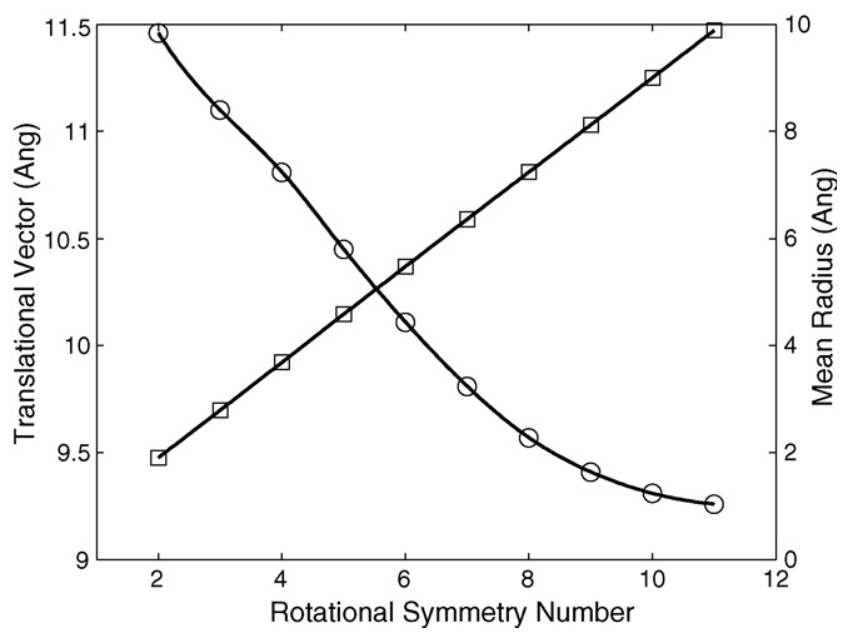

Fig. 3. The dependencies of translational vector (circle) and mean radius (square) of buckled CNT on the rotational symmetry. 
Table 3

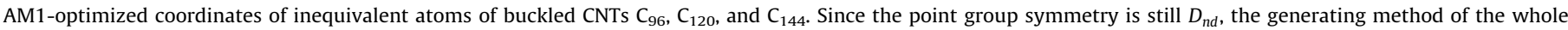
molecule is the same as the one for torus case described in text. The translational vectors (Tv) are listed in Table 4 .

\begin{tabular}{|c|c|c|c|c|c|c|c|c|c|}
\hline \multirow[t]{2}{*}{ Atom } & \multicolumn{3}{|l|}{$\mathrm{C}_{96}$} & \multicolumn{3}{|l|}{$C_{120}$} & \multicolumn{3}{|l|}{$C_{144}$} \\
\hline & $x$ & $y$ & $z$ & $x$ & $y$ & $z$ & $x$ & $y$ & $z$ \\
\hline 1 & 0.680 & -2.583 & 0 & 0.717 & -3.326 & & 0.721 & -4.041 & 0 \\
\hline 2 & 1.517 & -2.512 & 1.189 & 1.563 & -3.358 & 1.168 & 1.609 & -4.213 & 1.131 \\
\hline 3 & 1.290 & -3.362 & 2.328 & 1.297 & -4.296 & 2.241 & 1.306 & -5.229 & 2.122 \\
\hline 4 & 0 & -3.860 & 2.809 & 0 & -4.790 & 2.700 & 0 & -5.718 & 2.562 \\
\hline 5 & -4.112 & -0.722 & 6.336 & -4.632 & -2.266 & 6.179 & -4.907 & -3.665 & 6.006 \\
\hline 6 & 1.141 & -4.432 & 4.981 & 1.125 & -5.508 & 4.838 & 1.108 & -6.542 & 4.675 \\
\hline 7 & 0 & -4.460 & 4.083 & 0 & -5.481 & 3.930 & 0 & -6.472 & 3.756 \\
\hline$T v$ & 0 & & 10.81 & 0 & 0 & 10.45 & 0 & 0 & 10.11 \\
\hline
\end{tabular}

Table 4

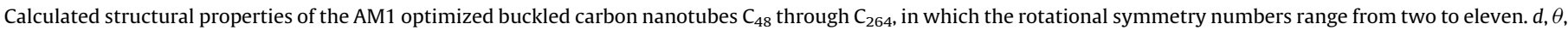

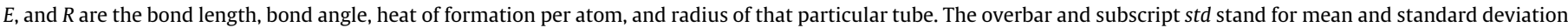
values, respectively. The translational vector $(T v)$ is parallel to $z$-axis.

\begin{tabular}{|c|c|c|c|c|c|c|c|}
\hline & $\bar{d}(\AA)$ & $d_{\text {std }}(\AA)$ & $\bar{\theta}\left({ }^{\circ}\right)$ & $\theta_{\text {std }}\left({ }^{\circ}\right)$ & $E(\mathrm{eV})$ & $T v(\AA)$ & $(\AA)$ \\
\hline$C_{48}$ & 1.444 & 0.033 & 115.8 & 5.2 & 0.97 & 1.91 & 11.46 \\
\hline$C_{72}$ & 1.434 & 0.027 & 117.7 & 4.9 & 0.61 & 2.79 & 11.10 \\
\hline$C_{96}$ & 1.433 & 0.034 & 118.3 & 5.6 & 0.53 & 3.69 & 10.81 \\
\hline$C_{120}$ & 1.432 & 0.029 & 118.4 & 5.9 & 0.53 & 4.59 & 10.45 \\
\hline$C_{144}$ & 1.433 & 0.031 & 118.4 & 6.5 & 0.56 & 5.48 & 10.11 \\
\hline$C_{168}$ & 1.435 & 0.034 & 118.3 & 7.1 & 0.62 & 6.36 & 9.81 \\
\hline$C_{192}$ & 1.436 & 0.037 & 118.2 & 7.7 & 0.68 & 7.24 & 9.57 \\
\hline$C_{216}$ & 1.439 & 0.041 & 118.1 & 8.3 & 0.75 & 8.12 & 9.41 \\
\hline$C_{240}$ & 1.441 & 0.045 & 118.0 & 8.9 & 0.82 & 9.00 & 9.31 \\
\hline$C_{264}$ & 1.443 & 0.050 & 118.0 & 9.5 & 0.88 & 9.89 & 9.26 \\
\hline
\end{tabular}

parent TCNT. Here we also give a brief examination of this process on the particular TCNTs discussed in the previous section. As shown in Fig. 4 , starting with the $D_{6 d}$ TCNT $C_{144}$ shown in Fig. 4(a), we shift the atoms in the outer-rim horizontally so that one of the neighbors of these atoms are switched from the originally bonded atom to the second-nearest neighbors (Fig. 4(b)). And the pentagons at the outer-rim pair up and come in contact with

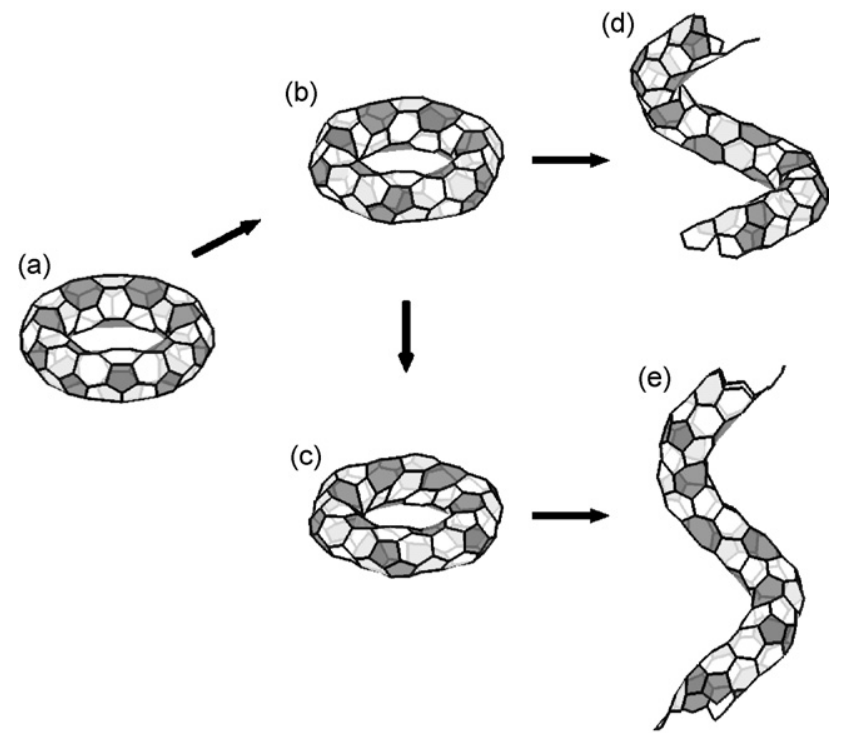

Fig. 4. Formation of HCNTs from the distortion of parent TCNT. (a) $D_{6 d} C_{144}$ parent buckytorus. (b) The outer-rim atoms of the buckytorus are shifted so that pentagons pair up and the reflection symmetry is destroyed. This molecule is considered to be highly strained and is denoted as $S h=1$. (c) Successive shifting operation is carried on to the TCNT shown in (b). This molecule is even more strained and is denoted as $S h=2$. (d) Dissecting the TCNT shown in (b), so the strain energy is released and resulting in an HCNT. (e) The HCNT corresponds to dissecting the TCNT shown in (c). The pitch angle, or the helicity, of the HCNT is larger than the one shown in (d). one another, so the analogy with $\mathrm{C}_{60}$ and $\mathrm{C}_{168}$ is destroyed. The resulting molecule is highly strained since all chemical bonds in this molecule (Fig. 4(b)) suffer considerable distortion. However, this strain can be greatly reduced by dissecting the TCNT at any longitude, which will allow the molecule to coil up to form an HCNT as shown in Fig. 4(d). We define the shifting parameter of this HCNT to be unity $(S h=1)$. Continuing the shifting operation with one more unit will lead to an even more strained molecule as shown in Fig. 4(c). Intuitively the pitch angle of the resulting HCNT generated by dissecting this transformed TCNT $(S h=2$, shown in Fig. 4(e)) is larger than the previous case with $S h=1$.

In general, once the molecular graph for a TCNT is drawn on a rectangular parametric plane [46], the 3D coordinates of the atoms in the corresponding HCNT are given simply by

$$
\begin{aligned}
x(s, t) & =(R+r \cos s) \cos t+r \sin \theta \sin s \sin t y(s, t) \\
& =(R+r \cos s) \sin t-r \sin \theta \sin s \cos t z(s, t) \\
& =R \tan \theta t+r \cos \theta \sin s,
\end{aligned}
$$

where $s$ and $t$ are the coordinates of the atoms on the parametric plane, $R$ the radius of the central helical curve, $r$ the radius of the tube around the central curve, and $\theta$ the pitch angle. Here the curve of constant $t$ corresponds to the circumference of the tube. Hence the curve with $s=0$ and varying $t$ corresponds to the middle line between the heptagons, and similarly, the curve with $s=\pi$ and varying $t$ corresponds to the middle line between the pentagons. When the shifting parameter $S h$ and the circumference of the tube $g$ are given, we found that the pitch angle $\theta$ is roughly given by $\tan \theta \propto S h / g$.

When the shifting parameter coincides with the length of a rotational unit cell $(S h=3)$, the resulting structure satisfies again the requirement of analogy to $C_{60}$, i.e. all nonhexagons are separated purely by hexagons and every carbon atom belongs to a particular nonhexagon, as shown in Fig. 5(a). Similar to the triply periodic $C_{168}$, this one-dimensional helical structure also contains 24 carbon atoms per unit cell. 

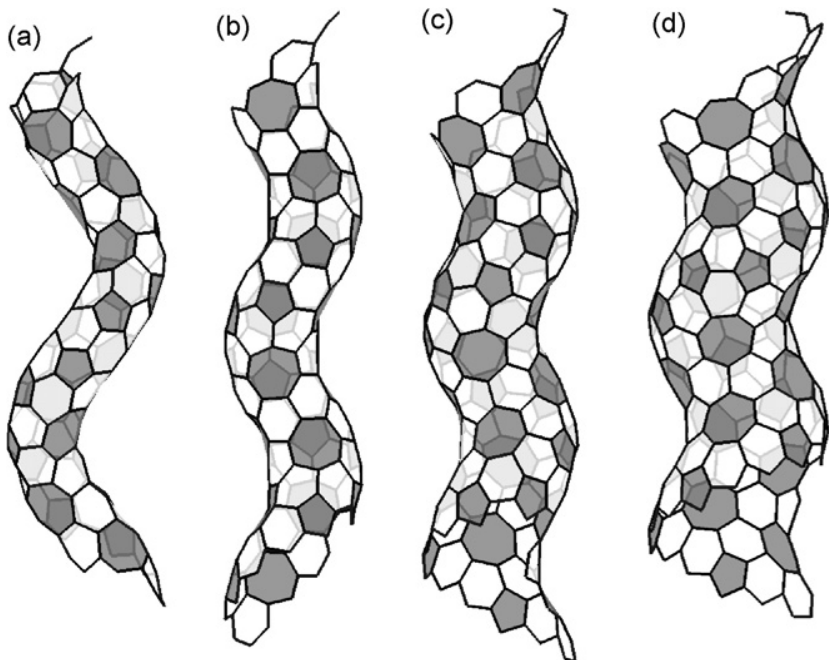

Fig. 5. Buckyhelices of higher shifting parameter $S h$. (a) $S h=3$, (b) $S h=6$, (c) $S h=9$, and (d) $S h=12$.

Naively, one might expect that the pitch angle for the HCNT derived from a particular TCNT should be a monotonic function of the shifting parameter $S h$. That is to say, the larger $S h$ is, the greater the pitch angle will be. One might predict that when $S h$ increases to a certain value, the pitch angle of the corresponding HCNT should become very close to $\pi / 2$, the pitch angle of a vertical straight line. However, it is known that the HCNTs with pitch angles larger than a critical value will be extremely unstable because the strong steric repulsion arising from the impenetrability among different parts of the HCNT when local curvature of the central axis is greater than the radius of tube $[47,48]$. But, a detailed calculation indicates that this situation does not occur even in the limit that the shifting parameter Sh becomes very large. Instead, the optimized geometry for the corresponding HCNT in the large Sh limit gradually adopts a unique conformation that is quite counter-intuitive, and the parameterization given by Eq. (1) does not hold in this limit.

For instance, as shown in Fig. 5(b)-(d), we have the optimized geometries for HCNTs with Sh twice, thrice, and four times of the length of a unit cell, respectively. One can see that the diameter of the corresponding HCNT increases as the shifting continues, while the pitch angle decreases as $S h$ increases. The dependence of pitch angle and tube radius on $S h$ is shown in Fig. 6. In this particular case, the pitch angle quickly reaches its maximum at $S h=3$ and

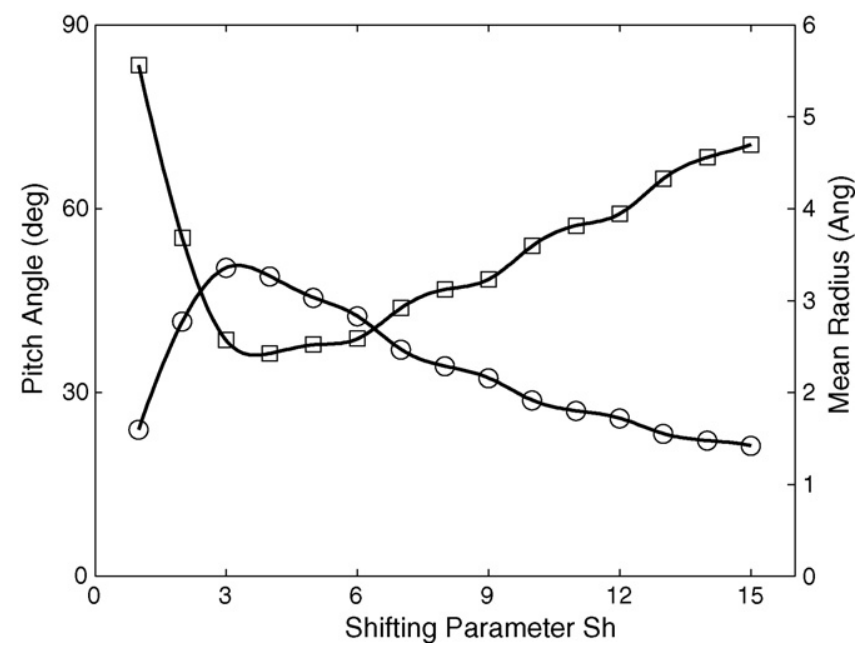

Fig. 6. Pitch angle (circle) and mean radius (square) as functions of shifting parameter Sh.

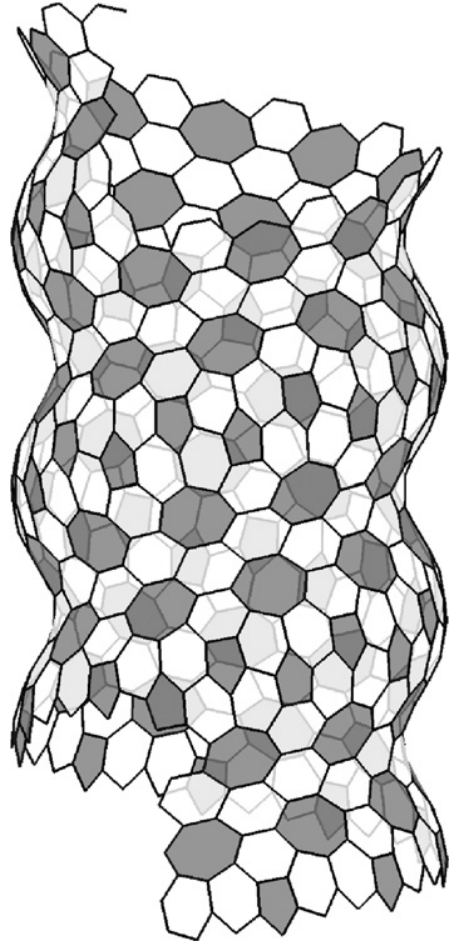

Fig. 7. Buckyhelix with $S h=30$.

decays slowly as $S h$ increases. On the contrary, the radii of the HCNT family show a minimum at $S h=3$ and gradually increases as $S h$ increases. If $S h$ is very large, the radius of the corresponding HCNT becomes linearly proportional to $S h$ and the pitch angle becomes inversely proportional to $S h$.

In the very large limit of $S h$, the corresponding HCNT is still a buckyhelix as shown in Fig. 7. Here we are not intended to discuss this trend in detail and leave it for the future investigation. We emphasize that by introducing the shifting operation to the buckytorus, a whole family of buckyhelices, infinite in number, can be constructed. Note that the buckled tube mentioned above with rotational symmetry number $n \rightarrow \infty$ is in fact corresponding to the transformed HCNT in the limit of $S h \rightarrow \infty$. But this implies that the surface of the tube is planar and atoms around the pentagons will be more crowded than ones around the heptagons, since they all possess zero Gaussian curvature.

\section{Conclusions}

In summary, toroidal, cylindrical, and helical analogs of $\mathrm{C}_{60}$ have been theoretically constructed. These structures possess positive and negative Gaussian curvature at the same time and contain equal numbers of pentagons and heptagons, which are compactly separated by hexagons. The cylindrical and helical buckyballs are singly periodic 1D graphitic structures. In particular, the cylindrical buckled tubes can be possible candidates for the product of coalescing arrays of $\mathrm{C}_{60}$ molecules. Physical properties such as electron transport and mechanical responses of these exotic graphitic structures are of theoretical interest and further investigation is needed.

\section{Acknowledgments}

This work was supported by a grant from NSC, Taiwan, ROC. We are grateful to Computer and Information Networking Center, National Taiwan University for the support of high-performance computing facilities. 


\section{References}

[1] S. Iijima, Helical microtubules of graphitic carbon, Nature 354 (1991) 56-58.

[2] D. Vanderbilt, J. Tersoff, Negative-curvature fullerene analog of C60, Phys. Rev. Lett. 68 (1992) 511-513.

[3] H. Terrones, A. Mackay, Triply periodic minimal-surfaces decorated with curved graphite, Chem. Phys. Lett. 207 (1993) 45-50.

[4] H. Terrones, A. Mackay, The geometry of hypothetical curved graphite structures, Carbon 30 (1992) 1251-1260.

[5] H. Terrones, A.L. Mackay, Negatively curved graphite and triply periodic minimalsurfaces, J. Math. Chem. 15 (1994) 183-195.

[6] A. Mackay, H. Terrones, Diamond from graphite, Nature 352 (1991) 762.

[7] T. Lenosky, X. Gonze, M. Teter, V. Elser, Energetics of negatively curved graphitic carbon, Nature 355 (1992) 333-335.

[8] P. Fowler, T. Pisanski, Leapfrog transformations and polyhedra of Clar type, J. Chem. Soc., Faraday Trans. 90 (19) (1994) 2865-2871.

[9] L. Liu, G.Y. Guo, C.S. Jayanthi, S.Y. Wu, Colossal paramagnetic moments in metallic carbon nanotori, Phys. Rev. Lett. 88 (2002) 217206.

[10] C.P. Liu, Zeeman effect on the electronic structure of carbon nanotori in a strong field, Int. J. Mod. Phys. B 22 (2008) 4845-4852.

[11] F.L. Shyu, Magneto-optical properties of carbon toroids: influence of geometry and magnetic field, Phys. Rev. B 72 (2005) 045424.

[12] C.P. Liu, N. Xu, Magnetic response of chiral carbon nanotori: the dependence of torus radius, Physica B-Cond. Matt. 403 (2008) 2884-2887.

[13] C.P. Liu, H.B. Chen, J.W. Ding, Magnetic response of carbon nanotori: the importance of curvature and disorder, J. Phys.-Cond. Matt. 20 (2008) 015206.

[14] F.L. Shyu, Influence of electric fields on magnetization of achiral carbon tori, Physica E-Low Dim. Syst. Nanostruct. 41 (2009) 537-542.

[15] N. Xu, J.W. Ding, H.B. Chen, M.M. Ma, Curvature and external electric field effects on the persistent current in chiral toroidal carbon nanotubes, Eur. Phys. J. B 67 (2009) 71-75.

[16] E.C. Kirby, R.B. Mallion, P. Pollak, Toroidal polyhexes, J. Chem. Soc., Faraday Trans. 89 (12) (1993) 2865-2871.

[17] D.J. Klein, Elemental benzenoids, J. Chem. Inf. Comput. Sci. 34 (1994) 453-459.

[18] H. Hosoya, Y. Okuma, Y. Tsukano, K. Nakada, Multilayered cyclic fence graphs: novel cubic graphs related to the graphite network, J. Chem. Inf. Comput. Sci. 35 (1995) 351-356.

[19] P.W. Fowler, P.E. John, H. Sachs, (3,6)-Cages, hexagonal toroidal cages, and their spectra, DIMACS Ser. Discrete Math. Theoret. Comput. Sci. 51 (2000) 139-174.

[20] M.V. Diudea, P.E. John, Covering polyhedral tori, MATCH Commun. Math. Comput. Chem. 44 (2001) 103-116.

[21] M.V. Diudea, B. Parv, E.C. Kirby, Azulenic tori, MATCH Commun. Math. Comput. Chem. 47 (2003) 53-70.

[22] M. Deza, P.W. Fowler, A. Rassat, K.M. Rogers, Fullerenes as tilings of surfaces, J. Chem. Inf. Comput. Sci. 40 (2000) 550-558.

[23] P.E. John, H. Sachs, Spectra of toroidal graphs, Discrete Math. 309 (2009) 2663-2681.

[24] J. Liu, H. Dai, J. Hafner, D. Colbert, R. Smalley, S. Tans, C. Dekker, Fullerene 'crop circles', Nature 385 (1997) 780-781.

[25] M. Ahlskog, E. Seynaeve, R. Vullers, C. Van Haesendonck, A. Fonseca, K. Hernadi, J. Nagy, Ring formations from catalytically synthesized carbon nanotubes, Chem. Phys. Lett. 300 (1999) 202-206.
[26] V. Meunier, P. Lambin, A.A. Lucas, Atomic and electronic structures of large and small carbon tori, Phys. Rev. B 57 (1998) 14886-14890.

[27] D.H. Oh, J.M. Park, K.S. Kim, Structures and electronic properties of small carbon nanotube tori, Phys. Rev. B 62 (2000) 1600-1603.

[28] S. Itoh, S. Ihara, J.I. Kitakami, Toroidal form of carbon C360, Phys. Rev. B 47 (1993) 1703-1704.

[29] S. Itoh, S. Ihara, Isomers of the toroidal forms of graphitic carbon, Phys. Rev. B 49 (1994) 13970-13974.

[30] J.E. Avron, J. Berger, Tiling rules for toroidal molecules, Phys. Rev. A 51 (1995) 1146-1149.

[31] B. Borstnik, D. Lukman, Molecular mechanics of toroidal carbon molecules, Chem. Phys. Lett. 228 (1994) 312-316.

[32] J. Berger, J. Avron, Classification Scheme for Toroidal Molecules, J. Chem. Soc., Faraday Trans. 91 (1995) 4037-4045.

[33] R. Tamura, M. Ikuta, T. Hirahara, M. Tsukada, Positive magnetic susceptibility in polygonal nanotube tori, Phys. Rev. B 71 (2005) 045418.

[34] S. Itoh, S. Ihara, Toroidal forms of graphitic carbon. II. Elongated tori, Phys. Rev. B 48 (1993) 8323-8328.

[35] C. Chuang, Y.-C. Fan, B.-Y. Jin, Generalized classification scheme of toroidal and helical carbon nanotubes, J. Chem. Inf. Model. 49 (2009) 361368.

[36] M.J.S. Dewar, E.G. Zoebisch, E.F. Healy, J.J.P. Stewart, Development and use of quantum mechanical molecular models. 76. am1: a new general purpose quantum mechanical molecular model, J. Am. Chem. Soc. 107 (1985) 39023909.

[37] J. Tersoff, Energies of fullerenes, Phys. Rev. B 46 (1992) 15546-15549.

[38] S. Hyde, M. OKeeffe, Elastic warping of graphitic carbon sheets: Relative energies of some fullerenes, schwarzites and buckytubes, Philos. Trans. Roy. Soc. Lond. A 354 (1996) 1999-2008.

[39] S. Ahmad, Continuum elastic model of fullerenes and the sphericity of the carbon onion shells, J. Chem. Phys. 116 (2002) 3396-3400.

[40] A. Šiber, R. Podgornik, Stability of elastic icosadeltahedral shells under uniform external pressure: application to viruses under osmotic pressure, Phys. Rev. E 79 (2009) 011919.

[41] I.A. Solov'yov, M. Mathew, A.V. Solov'yov, W. Greiner, Liquid surface model for carbon nanotube energetics, Phys. Rev. E 78 (2008) 051601.

[42] A. Šiber, Shapes and energies of giant icosahedral fullerenes-onset of ridge sharpening transition, Eur. Phys. J. B 53 (2006) 395-400.

[43] A. Šiber, Energies of $\mathrm{sp}(2)$ carbon shapes with pentagonal disclinations and elasticity theory, Nanotechnology 17 (2006) 3598-3606.

[44] J. Rodriguez-Manzo, F. Lopez-Urias, M. Terrones, H. Terrones, Magnetism in corrugated carbon nanotori: the importance of symmetry, defects, and negative curvature, Nano Lett. 4 (2004) 2179-2183.

[45] J.A. Rodriguez-Manzo, F. Lopez-Urias, M. Terrones, H. Terrones, Anomalous paramagnetism in doped carbon nanostructures, Small 3 (2007) 120-125.

[46] C. Chuang, Y.-C. Fan, B.-Y. Jin, Dual space approach to the classification of toroidal carbon nanotubes, J. Chem. Inf. Model. 49 (2009) 1679-1686.

[47] A. Maritan, C. Micheletti, A. Trovato, J.R. Banavar, Optimal shapes of compact strings, Nature 406 (2000) 287-290.

[48] S. Przybyl, P. Pieranski, Helical close packings of ideal ropes, Eur. Phys. J. E 68 (2001) 445-449. 